\title{
Psoriasis pustulosa generalizada de von Zumbusch. Reporte de un caso
}

\section{Generalized pustular psoriasis of von Zumbusch. A case report}

\author{
Lorenza M. Luengo-Fernández ${ }^{1 *}$, Uriel Pérez-Garciáa ${ }^{1}$, Andrés Tirado-Sánchez ${ }^{1}$, \\ María I. Arellano-Mendoza ${ }^{1}$, Rosa M. Ponce-Olivera ${ }^{1}$ y Patricia Mercadillo-Pérez ${ }^{2}$ \\ ${ }^{1}$ Servicio de Dermatología; ${ }^{2}$ Servicio de Dermatopatología. Hospital General de México Dr. Eduardo Liceaga, Ciudad de México, México
}

\begin{abstract}
Resumen
La psoriasis es una enfermedad inflamatoria crónica con una prevalencia mundial del 1-3\%. La psoriasis pustulosa generalizada es la forma más grave y representa solo el $1.8 \%$ de todos los casos de psoriasis. Su etiología es incierta, aunque se ha relacionado con el retiro repentino de corticosteroides sistémicos y tópicos. Se caracteriza por la presencia de pústulas estériles sobre piel eritematosa y dolorosa, y puede tener involucro sistémico importante. Presentamos el caso de un paciente con psoriasis pustulosa generalizada, sin compromiso sistémico grave, relacionada con el uso crónico e intermitente de betametasona en crema, con respuesta favorable a metotrexato.
\end{abstract}

Palabras clave: Psoriasis. Psoriasis pustulosa generalizada. Von Zumbusch. Corticosteroides.

\section{Abstract}

Psoriasis is a chronic inflammatory disease with a prevalence of 1-3\% worldwide. Generalized pustular psoriasis is the most severe clinical variant of psoriasis and represents only $1.8 \%$ of all psoriasis cases. Its etiology is uncertain, although it has been associated with the abrupt withdrawal of both systemic and/or topical corticosteroids. It is characterized by sterile pustules overlying painful erythematous skin and it may present with severe systemic involvement. We here present a case of generalized pustular psoriasis in a male with no severe systemic involvement, related to irregular and interrupted use of betamethasone cream, who responded favorably to methotrexate.

Keywords: Psoriasis. Generalized pustular psoriasis. von Zumbusch. Corticosteroids.

\section{Introducción}

La psoriasis es una enfermedad inflamatoria crónica, con una prevalencia reportada del 1-3\% en Europa y los Estados Unidos de América ${ }^{1}$. En México representa aproximadamente el $2 \%$ de la consulta dermatológica². Puede presentarse a cualquier edad, pero tiene una distribución bimodal entre los 15 y 20 años y entre los 55 y 60 años $^{1}$. La psoriasis pustulosa generalizada (PPG) representa menos del $1 \%$ de las formas graves de psoriasis².

La PPG se clasifica en formas localizadas (psoriasis pustulosa palmoplantar y acrodermatitis continua de Hallopeau) y forma generalizada (PPG de von Zumbusch).
Correspondencia:

*Lorenza M. Luengo-Fernández

E-mail: lore_3490@ hotmail.com 0210-5187/(O 2021 Colegio Ibero Latinoamericano de Dermatología A.C. (CILAD). Publicado por Permanyer. Este es un artículo open access bajo la licencia CC BY-NC-ND (http://creativecommons.org/licenses/by-nc-nd/4.0/).
Disponible en internet: 28-01-2022 Med Cutan Iber Lat Am. 2022;50(1):43-47 wir.MedinaCutanealLA.com 
Esta última fue descrita por primera vez en 1910 por von Zumbusch. La PPG que se desarrolla durante el embarazo se denomina impétigo herpetiforme. La psoriasis pustulosa juvenil es un subtipo poco frecuente de PPG que ocurre en menores de 18 años ${ }^{3-5}$.

La etiología de la psoriasis pustulosa es desconocida. Se ha relacionado con el uso de medicamentos, que incluyen terbinafina, ramipril, rituximab, clopidogrel, amoxicilina, esteroides tópicos y sistémicos, antiinflamatorios no esteroideos, ácido acetilsalicílico, sulfonamidas, litio, morfina, alquitranados, yoduro de potasio, progestinas e hidroxicloroquina. El retiro repentino de los corticosteroides (tanto tópicos como sistémicos) y de ciclosporina se ha relacionado frecuentemente con la PPG. En pacientes con antecedentes familiares de psoriasis o mutación homocigota de IL36RN, la edad de inicio es más temprana ${ }^{3,5}$.

La PPG se caracteriza por pústulas estériles de 2-3 mm, ocasionalmente dolorosas, que pueden asentarse sobre placas eritematoescamosas o desarrollarse en los bordes de las placas activas. Los pacientes presentan adicionalmente fiebre alta, malestar general, astenia y adinamia; en los estudios de laboratorio con frecuencia presentan leucocitosis con neutrofília, eosinofilia, proteína $C$ reactiva elevada, hipocalcemia e hipoalbuminemia ${ }^{3,5}$.

Las manifestaciones extracutáneas incluyen colestasis, colangitis, dolor epigástrico, artritis, neumonitis intersticial, lesiones orales como lengua geográfica y queilitis, lesión renal aguda y otitis media. Además, las lesiones pueden complicarse con infecciones bacterianas, como impétigo secundario ${ }^{3,5}$.

El diagnóstico de la PPG es clínico con confirmación histopatológica. El Consenso Europeo sobre los Fenotipos de Psoriasis Pustulosa ${ }^{6}$ define la PPG como pústulas estériles primarias, macroscópicamente visibles sobre la piel (respetando las palmas y las plantas), en ocasiones con síntomas de respuesta inflamatoria sistémica. Se excluyen los casos en que las pústulas se localizan únicamente sobre placas psoriásicas. El diagnóstico es mucho más probable en casos de repetición o de duración mayor de 3 meses $^{6}$.

Los hallazgos histopatológicos incluyen epidermis con paraqueratosis y acantosis, e infiltrado de células mononucleares en la dermis superficial con exocitosis de neutrófilos formando colecciones en el estrato córneo (microabscesos de Munro-Sabouraud) ${ }^{7-10}$ y subcórneas (macropústulas espongiformes de Kogoj) ${ }^{11}$.

No existe un tratamiento estandarizado para la PPG. Debido a la escasez de los casos y su naturaleza recurrente-remitente, se dificulta el desarrollo de ensayos clínicos controlados aleatorizados para investigar la eficacia y la seguridad de los diferentes esquemas disponibles $^{7-10,12}$. Presentamos un caso de PPG tratado exitosamente con metotrexato como tratamiento de primera línea.

\section{Caso clínico}

Varón de 36 años, con antecedente de psoriasis en placas de 6 años de evolución, tratada durante tiempo indefinido e intermitente con crema combinada con betametasona, clotrimazol y gentamicina. Seis días previos a su consulta presentó pústulas en el cuello, el tronco y ambas extremidades superiores, por lo que acudió a un médico facultativo que le indicó azitromicina, $500 \mathrm{mg} / 12 \mathrm{~h}$ por vía oral, y suspender el tratamiento con la crema combinada. El cuadro clínico se exacerbó 48 horas después, con eritema generalizado y aumento del número de pústulas, agregándose prurito intenso y malestar general, por lo que suspende el tratamiento con azitromicina y se indica deflazacort, $6 \mathrm{mg} / 8 \mathrm{~h}$ por vía oral, sin mejoría de la dermatosis en las siguientes 48 horas. Acudió a consulta externa de nuestro servicio para valoración. En la exploración física presentaba una dermatosis generalizada que respetaba las mucosas, constituida por placas eritematoescamosas con abundante escama gruesa amarillenta, untuosa, en algunos sitios de aspecto yesoso (Fig. 1). En el tronco y en las extremidades superiores e inferiores presentaba, sobre las placas eritematoescamosas y fuera de ellas, múltiples pústulas no foliculares de 3-5 mm (Fig. 2). Ambas palmas y plantas en menor grado, con escama gruesa amarillenta y fisuras.

El paciente negó artralgias y no se encontraron signos de artritis ni alteración en los signos vitales. Los estudios de laboratorio reportaron leucocitos $9.8 \times 10^{3} / \mu \mathrm{l}$, hemoglobina $14.8 \mathrm{~g} / \mathrm{l}$, plaquetas $276 \times 10^{3} / \mu \mathrm{l}$, creatinina $1.07 \mathrm{mg} / \mathrm{dl}$, ácido úrico $8.0 \mathrm{mg} / \mathrm{dl}$, glucosa $102 \mathrm{mg} / \mathrm{dl}$, colesterol total $134 \mathrm{mg} / \mathrm{dl}$, triglicéridos $180 \mathrm{mg} / \mathrm{dl}$, aspartato aminotransferasa $64 \mathrm{U} / \mathrm{I}$, alanina aminotransferasa $76 \mathrm{U} / \mathrm{l}$, gamma-glutamil transferasa $53 \mathrm{U} / \mathrm{l}$ y proteína C reactiva $179 \mathrm{mg} / \mathrm{dl}$. Se realizó biopsia de piel, la cual reportó hiperqueratosis laminar con microabscesos de Munro-Sabouraud, epidermis con hiperplasia psoriasiforme moderada, agranulosis, elongamiento de las crestas epidérmicas, espongiosis leve y exocitosis con formación de múltiples pústulas y abscesos de neutrófilos. En la dermis papilar se observaron capilares dilatados, y en la dermis reticular superficial un infiltrado inflamatorio perivascular leve de tipo 


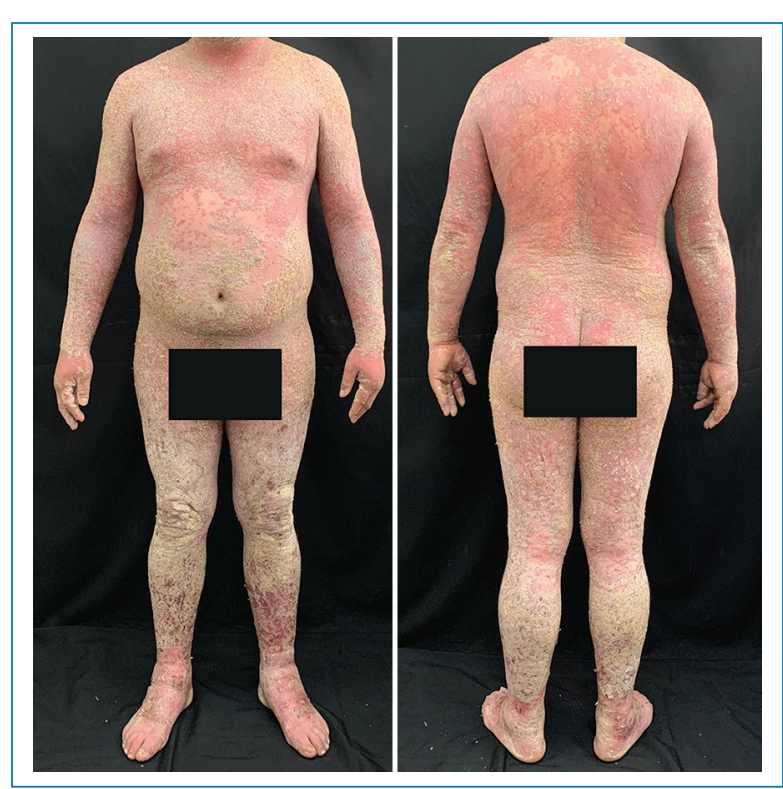

Figura 1. Dermatosis generalizada constituida por placas con intenso eritema, abundante escama gruesa y amarillenta, que en algunos sitios es de aspecto yesoso.

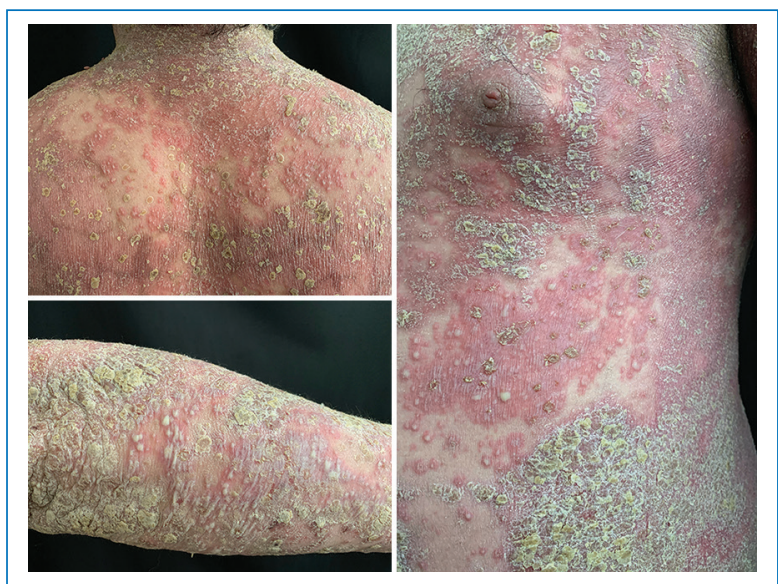

Figura 2. Múltiples pústulas sobre una base eritematosa en la cara posterior del tronco (tercio superior) y anterior (tercio medio), y en la cara posterior del antebrazo derecho.

linfohistiocitario (Fig. 3). Se integró un diagnóstico final de PPG con eritrodermia psoriásica y se indicó metotrexato, $10 \mathrm{mg}$ semanales, con una mejoría clínica significativa a las 5 semanas de alrededor del 80\% (Fig. 4) y persistencia de placas eritematoescamosas en el tronco y las extremidades inferiores, por lo que se decide aumentar la dosis de metotrexato a $12.5 \mathrm{mg}$ semanales, con evolución favorable.

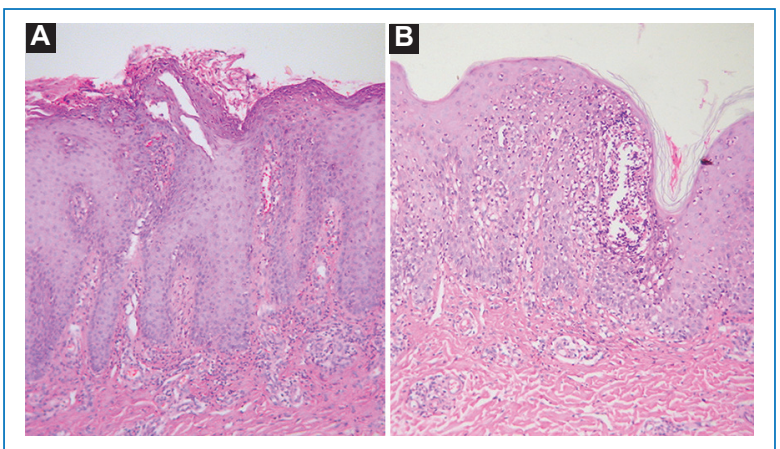

Figura 3. Estudio histopatológico con tinción de hematoxilina-eosina. A: estrato córneo con restos celulares de neutrófilos (microabsceso de Munro), hipogranulosis y acantosis regular de tipo psoriasiforme, dilatación de capilares de la dermis superficial con extravasación de eritrocitos e infiltrado inflamatorio perivascular compuesto por neutrófilos. B: acercamiento de la capa espinosa en el que se observa una pústula espongiforme con abundantes neutrófilos en su interior (pústula de Kogoj).

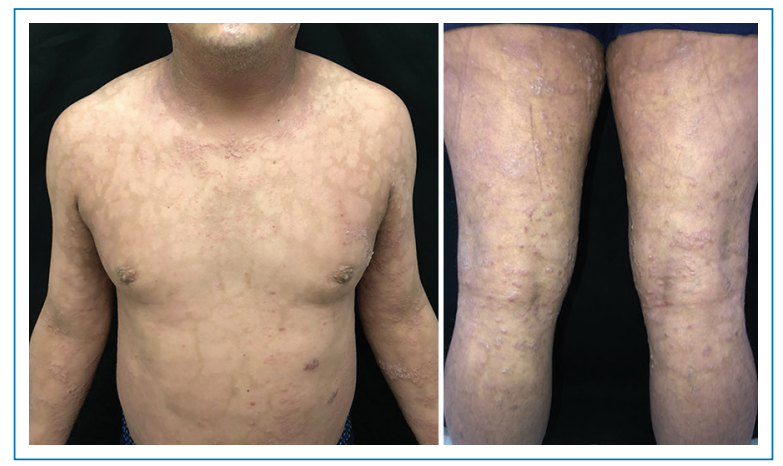

Figura 4. Evolución tras 5 semanas de tratamiento con metotrexato.

\section{Discusión}

La PPG es una variante poco frecuente de la psoriasis. Un análisis epidemiológico japonés de 2018 mostró que la PPG representa solo el $1.8 \%$ de todos los tipos clínicos de psoriasis, y en la población pediátrica del $0.6 \%$ al $7 \%$ de todos los casos de psoriasis ${ }^{3,13}$.

Las guías japonesas de $2018^{13}$ proponen hacer el diagnóstico definitivo de PPG cuando están presentes los cuatro criterios siguientes, y se sospecha cuando son positivos dos o tres criterios: a) síntomas sistémicos como fiebre y fatiga; b) eritema extenso o generalizado acompañado de múltiples pústulas estériles; c) pústulas subcórneas neutrofílicas (pústulas espongiformes de 
Tabla 1. Diferencias entre la psoriasis pustulosa generalizada y la pustulosis exantemática generalizada aguda

\begin{tabular}{|c|c|c|}
\hline & PPG & PEGA \\
\hline Factores precipitantes & $\begin{array}{l}\text { Infecciones, estrés, } \\
\text { corticosteroides (tratamiento o retiro } \\
\text { abrupto) }\end{array}$ & $\begin{array}{l}\text { Medicamento de reciente administración e } \\
\text { infecciones }\end{array}$ \\
\hline $\begin{array}{l}\text { Administración reciente de } \\
\text { medicamentos }\end{array}$ & Puede o no estar presente & $\begin{array}{l}\text { Gran mayoría de los casos } \\
\text { Antibióticos vía oral } 90 \%\end{array}$ \\
\hline $\begin{array}{l}\text { Antecedente personal o familiar } \\
\text { de psoriasis en placas }\end{array}$ & Presente en muchos casos & Raro \\
\hline Mutación en IL36RN & Presente & Presente \\
\hline Evolución & $\begin{array}{l}\text { Inicio insidioso, con episodios de larga } \\
\text { evolución y recurrencias }\end{array}$ & $\begin{array}{l}\text { Inicio abrupto y suele ser de corta evolución, sin } \\
\text { recurrencias }\end{array}$ \\
\hline Hallazgos clínicos & $\begin{array}{l}\text { Pústulas no foliculares y estériles sobre } \\
\text { placas eritematoescamosas o piel } \\
\text { eritematosa } \\
\text { Puede persistir semanas o meses }\end{array}$ & $\begin{array}{l}\text { Numerosa pústulas no foliculares y estériles sobre } \\
\text { base eritematosa } \\
\text { Involución en 4-10 días, dejando una escama } \\
\text { característica }\end{array}$ \\
\hline Afección del estado general & Frecuente & Frecuente \\
\hline Artritis & Presente $30 \%$ & Raro \\
\hline $\begin{array}{l}\text { Otras lesiones en piel } 0 \\
\text { mucosas }\end{array}$ & $\begin{array}{l}\text { Alteraciones ungueales, queilitis, lengua } \\
\text { geográfica y conjuntivitis }\end{array}$ & $\begin{array}{l}\text { Edema facial, vesículas, púrpura y lesiones tipo } \\
\text { eritema multiforme }\end{array}$ \\
\hline Histología & $\begin{array}{l}\text { Paraqueratosis, acantosis psoriasiforme, } \\
\text { microabscesos de neutrófilos en capa } \\
\text { córnea, pústulas espongiformes } \\
\text { subcórneas o intraepiteliales (Kogoj), } \\
\text { infiltrado neutrofílico en dermis papilar }\end{array}$ & $\begin{array}{l}\text { Pústulas espongiformes subcórneas o } \\
\text { intraepiteliales, edema en dermis papilar, infiltrado } \\
\text { perivascular neutrofílico y eosinofílico } \\
\text { En algunos casos queratinocitos necróticos y } \\
\text { vasculitis leucocitoclástica }\end{array}$ \\
\hline
\end{tabular}

IL36RN: gen antagonista del receptor de interleucina 36; PEGA: pustulosis exantemática generalizada aguda; PPG: psoriasis pustulosa generalizada.

Kogoj); y d) recurrencia del cuadro clínico y de los hallazgos histopatológicos mencionados ${ }^{13}$. De acuerdo con estos criterios, nuestro paciente se clasificó como sospecha de PPG, pero por los antecedentes, la correlación clínico-patológica y la adecuada respuesta al tratamiento de primera línea consideramos que puede definirse como PPG de aparición inicial.

Se han descrito múltiples desencadenantes de esta patología, como interrupción abrupta del tratamiento con esteroides ${ }^{14}$, infecciones, embarazo ${ }^{8}$, hipocalce$\mathrm{mia}^{15} \mathrm{e}$ inicio de terapia biológica, como ustekinumab ${ }^{16}$ 0 antagonistas del factor de necrosis tumoral (TNF) ${ }^{17}$. En nuestro caso consideramos que el paciente desarrolló la eritrodermia psoriásica por el uso irregular e intenso de betametasona; la abrupta interrupción del esteroide probablemente contribuyó al desarrollo de la PPG o pudo estar retrasando la aparición de pústulas.

Se debe hacer el diagnóstico diferencial con la pustulosis exantemática generalizada aguda, ya que clínicamente y por histopatología pueden ser indistinguibles. Para poder diferenciar ambos cuadros es útil la presencia de acantosis, característica de psoriasis y el antecedente de psoriasis, aunque se han reportado casos de PPG sin este antecedente ${ }^{10}$ (Tabla 1) ${ }^{18-22}$.

A pesar de no ser parte de los criterios diagnósticos, los hallazgos en los estudios de laboratorio (leucocitosis, elevación de la proteína $C$ reactiva, hipocalcemia e hipoalbuminemia) ayudan a determinar la gravedad de la enfermedad y las posibles complicaciones ${ }^{13}$. Nuestro paciente solo presentó valores elevados de proteína $\mathrm{C}$ reactiva, y por la ausencia de otros síntomas sistémicos no requirió manejo intrahospitalario.

Las complicaciones de la PGG van desde afección articular y oftálmica hasta otras que pueden comprometer el pronóstico de la enfermedad, como sepsis, insuficiencia respiratoria, colestasis inducida por colangitis neutrofílica y amiloidosis secundaria ${ }^{13,23}$, por lo que el diagnóstico precoz y el tratamiento oportuno son esenciales.

Como tratamiento de primera línea pueden utilizarse acitretina, ciclosporina o metotrexato. Los corticosteroides orales únicamente deben indicarse cuando las otras opciones no se encuentran disponibles o están 
contraindicadas $^{7,10}$. Como segunda línea, en adultos se pueden combinar tratamientos biológicos con no biológicos de primera línea. Entre los más empleados se encuentran los agentes anti-TNF como el infliximab, el adalimumab y el etanercept, los anti-IL-1 como el anakinra ${ }^{7}$, los anti-IL-17 como el secukinumab y el ixekizumab, y los anti-IL-12 e IL-23 como el ustekinumab ${ }^{12}$. Se ha descrito también, como segunda línea de tratamiento, la fototerapia PUVA. Los agentes tópicos pueden aplicarse como terapia adyuvante; los más utilizados son los corticosteroides, el calcipotrieno y el tacrolimus ${ }^{7,10}$.

\section{Conclusiones}

La importancia de este caso radica en que hay que tener siempre presentes las variantes clínicas poco comunes de la psoriasis, como la PPG, siendo esta una enfermedad grave que potencialmente puede comprometer la vida del paciente, además de ser de difícil diagnóstico (deben descartarse farmacodermias).

Debido a la extensión de la dermatosis es necesario monitorizar y tratar las complicaciones asociadas, como desequilibrio hidroelectrolítico, lesión renal aguda o procesos infecciosos que pueden complicar el pronóstico. En el caso presentado se observó una buena respuesta clínica al tratamiento sistémico de primera línea y la ausencia de complicaciones, principalmente por el diagnóstico temprano y el tratamiento oportuno.

\section{Financiamiento}

No se ha recibido financiamiento para la realización de este artículo.

\section{Conflicto de intereses}

Los autores declaran que no existe conflicto de intereses.

\section{Responsabilidades éticas}

Protección de personas y animales. Los autores declaran que para esta investigación no se han realizado experimentos en seres humanos ni en animales.

Confidencialidad de los datos. Los autores declaran que han seguido los protocolos de su centro de trabajo sobre la publicación de datos de pacientes.

Derecho a la privacidad y consentimiento informado. Los autores han obtenido el consentimiento informado de los pacientes y/o sujetos referidos en el artículo. Este documento obra en poder del autor de correspondencia.

\section{Bibliografía}

1. Brandon A, Mufti A, Gary Sibbald R. Diagnosis and management of cutaneous psoriasis: a review. Adv Skin Wound Care. 2019;32:58-69.

2. Chanussot $C$, Arenas R. Psoriasis. Estudio descriptivo y comorbilidades en 114 pacientes. Dermatol Cosmet Med Quirur. 2015;13:20-3.

3. Ly K, Beck KM, Smith MP, Thibodeaux Q, Bhutani T. Diagnosis and screening of patients with generalized pustular psoriasis. Psoriasis (Auckl). 2019:9:37-42.

4. Bachelez H. Pustular psoriasis and related pustular skin diseases. $\mathrm{Br} \mathrm{J}$ Dermatol. 2018;178:614-8.

5. Hoegler KM, John AM, Handler MZ, Schwatz RA. Generalized pustular psoriasis: a review and update on treatment. J Eur Acad Dermatol Venereol. 2018;32:1645-51

6. Navarini AA, Burden AD, Capon F, Mrowietz U, Puig L, Köks S, et al.; ERASPEN Network. European consensus statement on phenotypes of pustular psoriasis. J Eur Acad Dermatol Venereol. 2017;31:1792-9.

7. Benjegerdes KE, Hyde K, Kivelevitch D, Mansouri B. Pustular psoriasis: pathophysiology and current treatment perspectives. Psoriasis (Auckl). 2016;6:131-44.

8. Choon SE, Lai NM, Mohammad NA, Nanu NM, Tey KE, Chew SF. Clinical profile, morbidity, and outcome of adult-onset generalized pustular psoriasis: analysis of 102 cases seen in a tertiary hospital in Johor, Malaysia. Int J Dermatol. 2014;53:676-84.

9. Ortega A, Restrepo N, Rosero YS, Úsuga F, Correa LA, Marín ND, et al. Características epidemiológicas, clínicas e histopatológicas de pacientes con psoriasis y factores asociados con las formas vulgar y pustulosa. Dermatol Rev Mex. 2018:62:193-205.

10. Gooderham M, Van Voorhees A, Lebwohl M. An update on generalized pustular psoriasis. Expert Rev Clin Immunol. 2019;15:907-19.

11. Calonje E, Brenn T, Lazar A, McKee P. Spongiotic, psoriasiform and pustular dermatoses. En: Calonje E, Brenn T, Lazar A, McKee P, editores. McKee's pathology of the skin: with clinical correlations. $4^{\text {th }}$ ed. Edinburgh: Elsevier Saunders; 2012. p.180-218.

12. Saeki $H$, Nakagawa $H$, Nakajo $K$, Ishii $T$, Morisaki $Y$, Aoki $T$, et al. Efficacy and safety of ixekizumab treatment for Japanese patients with moderate to severe plaque psoriasis, erythrodermic psoriasis and generalized pustular psoriasis: results from a 52-week, open-label, phase 3 study (UNCOVER-J). J Dermatol. 2017;44:355-62.

13. Fujita H, Terui T, Hayama K, Akiyama M, Ikeda S, Mabuchi T, et al. Japanese Dermatological Association Guidelines Development Committee for the Guidelines for the Management and Treatment of Generalized Pustular Psoriasis. Japanese guidelines for the management and treatment of generalized pustular psoriasis: the new pathogenesis and treatment of GPP. J Dermatol. 2018;45:1235-70.

14. Aguayo S, Vélez J, Irigoyen E, Heredia M, Morocho P, Paredes M, et al. Psoriasis pustulosa generalizada de Von Zumbusch: reporte de un caso. Pro Sci. 2019;3:6-9.

15. Gusmão C, De Assis L, Góes P, Rosa F, Nunes V, Gusmão I, et al. A case of acute generalized pustular psoriasis of von Zumbusch triggered by hypocalcemia. Case Rep Dermatol. 2015;7:345-51.

16. Dai $Y$, Chen $C$. Flare-up of pustular psoriasis after ustekinumab therapy: case report and literature review. Dermatol Sin. 2018;36:222-5.

17. Collamer A, Battafarano D. Psoriatic skin lesions induced by tumor necrosis factor antagonist therapy: clinical features and possible immunopathogenesis. Semin Arthritis Rheum. 2010;40:233-40.

18. Kardaun SH, Kuiper H, Fidler V, Jonkman MF. The histopathological spectrum of acute generalized exanthematous pustulosis (AGEP) and its differentiation from generalized pustular psoriasis. J Cutan Pathol. 2010;37:1220-9

19. Navarini AA, Simpson MA, Borradori L, Yawalkar N, Schlapbach C. Homozygous missense mutation in IL36RN in generalized pustular dermatosis with intraoral involvement compatible with both AGEP and generalized pustular psoriasis. JAMA Dermatol. 2015;151:452-3.

20. Feldmeyer L, Heidemeyer K, Yawalkar N. Acute generalized exanthematous pustulosis: pathogenesis, genetic background, clinical variants and therapy. Int J Mol Sci. 2016;17:1214.

21. Syed T, Abdullah A, Mubasher M, Yousaf Z, Mohamed M, Alweis R. Acute generalized exanthematous pustulosis with multiple organ failure. Case Rep Dermatol. 2021;13:47-53

22. Jurado F, Cardona MA, Ramos A, Rossiere NL, Ríos Z. Pustulosis exantemática generalizada aguda. Presentación de un caso y revisión de la literatura. Rev Cent Dermatol Pascua. 2016;25:27-31.

23. Zelickson B, Muller S. Generalized pustular psoriasis. A review of 63 cases. Arch Dermatol. 1991;127:1339-45. 